\title{
Mechanism of Softening Effect of Fabric Softener.
}

\author{
Takako Igarashi $^{1,2} \&$ Koichi Nakamura ${ }^{2}$ \\ ${ }^{1}$ Household research Laboratories, Kao Corporation, Japan \\ ${ }^{2}$ Material Science Research Laboratories, Kao Corporation, Japan \\ Correspondence: Takako Igarashi, Household research Laboratories, Kao Corporation, Japan. E-mail: \\ igarashi.takako@kao.com
}

Received: December 7, 2018

Accepted: December 27, 2018

Online Published: December 31, 2018

doi: $10.5539 /$ jmsr.v8n1p35

URL: https://doi.org/10.5539/jmsr.v8n1p35

\begin{abstract}
The mechanism of softening effect for fabric softeners has been explained as lowering of friction between the fibers. This explanation, however, has not been verified. The trend data of B-value of KES-FB 2 and the result of perfect drying cotton threads indicate that the increase of hardness of cotton threads after the process of wetting by water and drying is caused by the cross-linking by the bound water between the cotton fibers. Thus, the softening effect of fabric softeners can mainly be discussed as the prevention of the formation of this cross-linkage.
\end{abstract}

Keywords: fabric, cotton, softener, friction, hydrogen bonding, bound water, non-freezing type of water, cross-linkage

\section{Introduction}

The utility of cationic surfactants as household softeners was discovered incidentally in the course of a research on direct-dye water-resistant conditioning agents for cotton fibers in the 1930s (Evance, 1969). Household softeners were first sold in the US in 1955 (Egan, 1978; Puchta,1984) and, in 1962, Kao Softer was introduced to Japanese market as the first softener with the dual action of fabric softening and imparting anti-static properties (Minegishi, 1977). This product subsequently became established as a laundry consumer product indispensable and essential to our daily lives; it currently holds a domestic market share of approximately 100 billion JPY (Japan Nichiyohin-Keshohin Shimbun,2013). If we look at the types of softeners used globally, they can be roughly divided into liquid-type softeners and sheet-type softeners (used in dryers). However, the use of sheet-type softeners is limited to Western countries where drum-type washer-dryers are widely used, whereas only liquid-type softeners are used in Japan. The functionality of softeners as the appealing message to the consumers has diversified in recent years, with the market overflowing with a large number of products, are focused on fragrance, deodorizing, and/or antibacterial qualities. Nonetheless, the primary function - value for consumers originally provided by softeners is still the softening of clothes, which remains unchanged.

Clothing fibers harden and become stiff after repeated routine washing and natural drying, but adding a small amount of softener during the laundry rinse cycle can easily result in the condition that makes the clothes feel soft to the touch. A type of fiber for which this effect is markedly notable is cotton. A large number of varied clothing materials with superior appearance, texture properties, and functionality are in circulation in the apparel industry owing to the recent spread of synthetic fibers. However, cotton fiber still occupies approximately $40 \%$ of the global fiber production, fulfilling its role as a material central to the clothing aspects of the lives of humans, as it has done since prehistoric times.

Therefore, this study summarizes existing researches on the essence of softening, focusing on cotton fiber, to determine how cotton is softened.

\section{Background of Previous Research}

The main bases of household softeners distributed on today's market can be broadly divided into di-long-chain alkyl-type cationic surfactants (Iwazawa, Umezawa, Sawada, \& Tsuji, 2001) and silicon (Harberder \& Bereck, 2002; Miyasaka, 2004; Egawa, 2008), but almost all belong to the former category. The softness derived from the use of cationic surfactants has been generally explained in relation to inter-fiber friction. That is, as shown in Figure 1, when softeners are used on fibers in water, the cationic vesicles made of softener molecules are adsorbed 
via electrostatic interactions to the negatively charged surfaces of the fibers; mono-layers on the fiber surface are thus formed during the drying process with the alkyl groups having low surface energy facing toward the air side, thereby reducing the friction between adjacent fibers. This lowered inter-fiber friction has been taken as the cause of softness (American Association of Textile Chemists and Colorists [AATCC], 1978; Okumura \& Yokoi, 1995; Seno \& Tsuji, 1995; Takeuchi, 1999; Katayama, 2003; Miyazaki, 2005; Mohammadi, 2006; Morita, 2007; Shimazaki \& Sasai, 2007; Abe \& Horiuchi, 2011). The grounds for this explanation are described below.

Adhesion of Vesicles

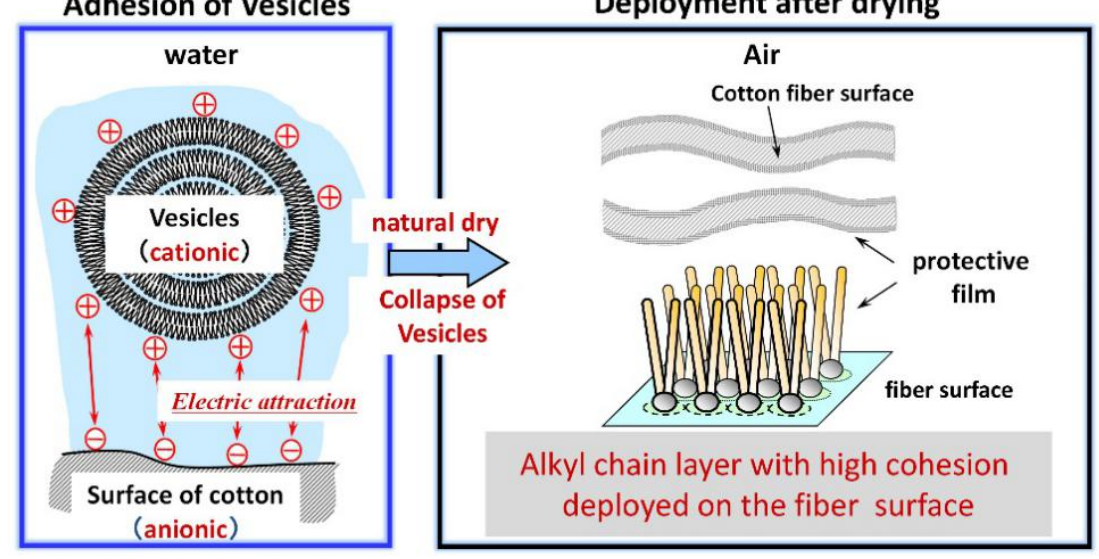

Figure 1. Conventional Explanation of the "Mechanism of Softening Effect"

First, it is known that softener exists in water as a phospholipid-like multi-layer film structure called as "vesicle", and because softeners have a cationic charge, it is thought that they are adsorbed onto the negatively charged surfaces of fibers through electrostatic interactions (Suzawa \& Yuzawa, 1966; Egan, 1978). The reason for the fiber surfaces having a negative charge is that the chemical structure of cotton fiber is composed of cellulose, part of which changes to a structure containing a carboxyl group through oxidation reactions (Buecking, Loetsch, \& Taeuber, 1984). The reason for this electrostatic-interaction-driven adsorption is thought to be an analogy caused by the surfactant itself behaving as a cationically-charged colloid in water.

However, there has been uncertainty associated with this mechanism, because the sequence of adsorption is thermodynamically unfavorable: in the case of cationic vesicle, adsorption is followed by the formation of multi-layers between the fibers and water by the cationic surfactants, which are sparingly soluble in water, together with subsequent formation of a mono-layers between the fibers and air when the clothe is dried. This process is quite complex.

In response to this, Cruzen (1995) argued that the hydrophobic properties (hydrophobic bonds) derived from the long-chain alkyl groups were the driving force of adsorption, given that a softener can be adsorbed not only onto cotton, but also onto other artificial fabric surfaces which does not have any meaningful electric charge. As an additional interesting information, Minegishi (1997) investigated the adsorption rate of softener into cotton fiber over time and reported that the time taken to reach adsorption equilibrium was heavily dependent on mechanical force. This information suggests that softener adsorption is strongly governed by fiber collision. Furthermore, in terms of the adsorbed state of the softener into the fabric surface, Okumura (1983) reported that softener is uniformly adsorbed, retaining its multi-layer particle shape, whereas Nakamura (1997) reported that the vesicles adsorbed into the fabric surface collapsed during the drying process, forming a circular shape with overlapping bi-layer membranes that then transitioned to an interdigitated structure with reduced thickness and finally becomes the mono-layer when this drying process ends with the alkyl-chains of the softening agent are exposed to air. Sakai et al. (2019) recently used atomic force microscopy to observe the adsorption on the surface of mica, and reported that the vesicles adsorbed by collision immediately expanded to form a double-layered molecular membrane. The adsorption state of the softener molecules was then maintained without loss of the molecules from the surface, because of the electrostatic interaction between the anionic part of the cotton surface and the cationic moiety of the softener molecule. Based on these information, even now, there are still a number of theories on the driving force behind the adsorption of softeners onto fibers and on the adsorption state, and we are not yet at a stage where this phenomenon can be definitively explained. 
$<$ Why fibers treated with softener become softer? $>$

As mentioned at the beginning of this article, the conventional or so-called "common theory" for the origin of this softness has been explained with relation to the phenomenon of inter-fiber friction. There have been a large number of reports since 1930 on attempts to capture the textile (texture) properties of fabric not only after softening, but also when applying stress-change using external forces in order to establish some link with the fiber's properties. It can be said that softeners emerged on the market with this era as the backdrop. The report by Evance (1969) is one of the notable review articles relating to softeners from this time and, in his report, he discussed that plasticizing and friction reduction were the main causes of fiber softening. The most effective agent for plasticizing cotton fibers is water, but the effectiveness of poly-alcohols and hygroscopic salt is also mentioned in his review. However, when using a cationic surfactant, there is no plasticizing (intensity change) of the fiber itself occurs and, considering that the agents used at that time did not thought to penetrate inside the single fibers, lubrication on the fabric surface fastened upon was considered as the only cause of the perceived softness. However, in this case, only proof-by-contradiction arguments have been made about this concept, and there is no assertive basis indicating that the reduction of surface friction is the direct cause of the fabric softening effect.

Specific attempts to link fiber friction properties with softness and to interpret the results have been carried out.

Larrat (1966) conducted a study using both a friction meter and Handle-o-Meter (a device used to evaluate the bending stiffness of textiles, which measures composite values for bending and sliding and was developed by Johnson \& Johnson and manufactured and sold by Thwing-Albert) and reported that he observed a reduction in friction with an increased perception of softness when using the friction meter, whereas he reported no correlation was found when using the Handle-o-Meter. In contrast to this, Röder (1953) and Olofsson (1950) reported a correlation between the perception of softness and reduced inter-fiber friction, and a relative correlation between the coefficient of static friction and the coefficient of dynamic friction, stating that having a small coefficient of static friction was important for softness. In other words, these information indicated that the perception of softness and the perception of squeaky feel are generated by differences in the "relative position" of static friction and dynamic friction. Given that the studies of Roeder and Olofsson were cited by a large number of subsequent softener-related reports, it can be assumed that these studies may have been the grounds for the establishment of "friction-reduction theory" on softening agents. However, the coefficient of friction correlating to the extent of softness does not always indicate a direct causal relationship. Motoyama \& Saiuchi (1961) touched upon the report made by Roeder and, although they affirmed the involvement of the friction phenomenon, they also pointed out through measurement results that not only the absolute measurement values of the coefficient of friction changes, but even the relative tendency can also changes. Therefore, they mentioned that agents that effectively reduce inter-fiber friction may not necessarily be effective for reducing the friction between skin and fiber, which is essential when considering the texture of the softener-treated clothe. Furthermore, in an aforementioned report, Crutzen (1995) also introduced the concept that good softeners do not necessarily effectively reduce the coefficient of friction. The authors measured the friction of yarns using a combination of a number of different methods, including using a $\mu$-meter, but were unable to confirm a trend of consistent reduction in static friction and dynamic friction when using softener.

Moreover, Sebastian, Bailey, Briscoe, and Tabor (1986) reported that the junction rupture force (JRF) value when a single thread is pulled from a plain-woven fabric, namely the static coefficient of friction generated between the fabric and the pulled thread, is a good indicator of softness. However, it was later clarified that this method not only measures simple frictional force values, but also simultaneously measures the adhesive force between fibers.

Conversely, by looking at fiber properties other than friction, Stock (1953) investigated on using a drape meter, which can be used to obtain an indicator of hardness, by measuring the extent to which fabric hang down from the round tester table under its own weight, whereas Motoyama and Saiuchi (1961) proposed the Clark's stiffness testing method, which is able to measure bending and stiffness. Hawarth (1964) conducted experiments after making a working hypothesis emphasizing the importance of fiber surface roughness and flexibility relating to friction in order to explain the perception of softness, and, as a result, stated that its relationship with softness could not be explained.

Within the flow of subsequent researches on texture of cloth, in 1972 the Textile Machinery Society of Japan proposed the measuring gauge of texture and the Standardization Research Committee proposed Kawabata's evaluation system (KES, 1982) as the standard methods for measuring texture of cloth. This popularized the quantification of cloth texture values, which was previously performed via subjective human evaluations. This method can calculate basic texture values (KOSHI: stiffness, NUMERI: slimy-ness, FUKURAMI: fullness \& softness, SHARI: crispness, HARI: anti-drape strength, KISHIMI: squeaky feel, SHINAYAKASA: flexibility, 
SOFUTOSA: soft feel) from 17 mechanical properties concerning tensile properties, bending properties, shearing properties, compression properties, surface properties, and thickness. Inoue, Sano, Uyama, and Niwa (1997) used this method to investigate the effect of softeners and reported an improvement in the texture related properties (NUMERI, FUKURAMI, SOFUTOSA, etc.) based on the increased shear properties, shear hysteresis, bending stiffness, bending hysteresis, and tensile resilience, as well as changes in the surface properties. However, this report does not present detailed reasons for the acquired results. The KES is widely and frequently used both in Japan and overseas, but it is a method developed for measuring sheer fabric, such as those used in men's suits. Thus, it has been known from the outset that it cannot always be used on any products, including products with piles such as towels.

Subsequently, Laghlin (1991), Farooq and Schramm (2009), and others stated that the best method for appropriately expressing soft feeling is to carry out sensory evaluations (subjective evaluations) by expert panelists, which had been used since the beginning of this field of research, touching upon the viewpoint that indicates the superiority of the human somatosensory sense. In this way, the softness of fabric is defined to be the sensations felt by the person feeling the fiber with bare hands, which is recognized during manipulation of the fabric, such as when performing compression and bending as one of the preference. Thus, at the current stage, we have not fully reached to the point where we can fully discuss so-called "softness".

\section{Mechanisms for the Production of New Softener Effects}

To counter the aforementioned situation, we conducted research from a different perspective of that of conventional methods in an attempt to understand the essential phenomenon that causes the feeling of stiffness at first and then softness which is realized when softener is used (ref. Igarashi, Nakamura, Morita \& Okamoto 2016). The details are described below.

Normally, various fiber processing agents are used in fiber manufacturing processes to improve operability during machining processes and get the finishing effects. Therefore, to remove these fiber processing agents, if a fully pre-laundered cotton towel is used and left to dry naturally after being wet, the resulting towel would be very stiff, just as if it had been starched. Generally, softeners are used to reduce this stiffness. On the other hand, when we replicated routine washing operations, allowing the towel to dry naturally after removing all the water and shaking it. These actions alone, without using softener, dramatically changed the stiffness of the towel, allowing for the arising of softness. The results obtained after reproducing this phenomenon using a bundle of cotton thread washed in solvent are shown in Figure 2. When we compared the results of Treatment A (leaving the bundle to dry naturally after washing with water) and Treatment B (leaving the bundle to dry naturally after shaking after removing the washing water), it is easy to appreciate the significant differences in the appearance of both thread bundles. As mentioned earlier, the thread bundle that underwent Treatment A was stiff, and thus it retained its horizontal shape without falling in the direction of gravity, whereas the thread bundle that underwent Treatment B hung down completely.

Treatment A

[wash $\rightarrow$ spin-dry $\rightarrow$ flutter $\rightarrow$ dry]

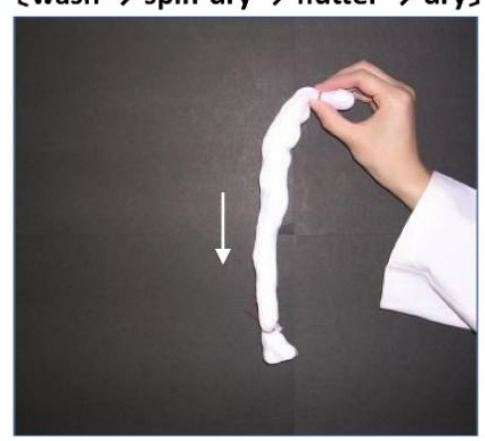

Treatment B [wash $\rightarrow$ natural-dry]

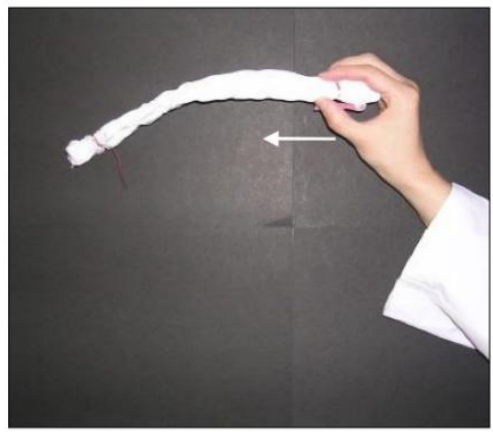

Figure 2. Appearance of Cotton thread Bundles (without Softener)

Thus, we focused on this phenomenon in which cotton fibers dried naturally from a wet state become extremely stiff, moving forward by searching for the mechanism that causes the appearance of the essential effects of softeners by scrutinizing the factors that cause stiffness, which is the opposite of softness. 
Almost all of the secondary wall, which accounts for the majority of scoured cotton, is comprised of micro-fibrils of regularly arranged cellulose molecules, and thus the presence of innumerable hydroxyl groups on the surface (Rollons \& Tripp, 1954) and simply removing water and shaking out a washed towel dynamically change the softness of the fabric. Based on this finding, we thought that the aforementioned stiffness phenomenon was derived from some kind of 3-dimensional bond network between single fibers. Therefore, we used a pure bending tester (KES-FB 2 , Kato Tech) to quantitatively interpret these bonds. We pre-treated cotton threads washed in solvent and treated the resulted threads with different softener concentrations, namely $0-0.3 \%$ o.w.f. (on the weight of the fabric), where $0.1 \%$ o.w.f. is the standard concentration for fabric softeners. The resulting threads (35 threads) were arranged so that they did not come into contact with each other and were fixed at both ends with double-sided tapes as shown in Figure 3. The threads were soaked with water and then ventilation-dried for two full days in the standard condition $\left(25^{\circ} \mathrm{C}, 60 \% \mathrm{RH}\right)$. Then, the pure bending tests were performed. These tests are carried out to measure the moment when the sample is bent to $270^{\circ}$, i.e., the B-value $\left(\mathrm{gf} \cdot \mathrm{cm}^{2} / \mathrm{cm}\right)$. The results obtained by repeating these tests are shown in Figure 4. As can be seen from these results, (1) the B-value is significantly reduced by the initial bending operation; (2) the B-value then converges to an almost constant value; (3) increasing the softener concentration reduces the B-value for both (1) and (2), but if excessive agent is used $(0.2-0.3 \%$ o.w.f.), the initial reduction in (1) is no longer seen and the value in (2) remains constant at a low value.
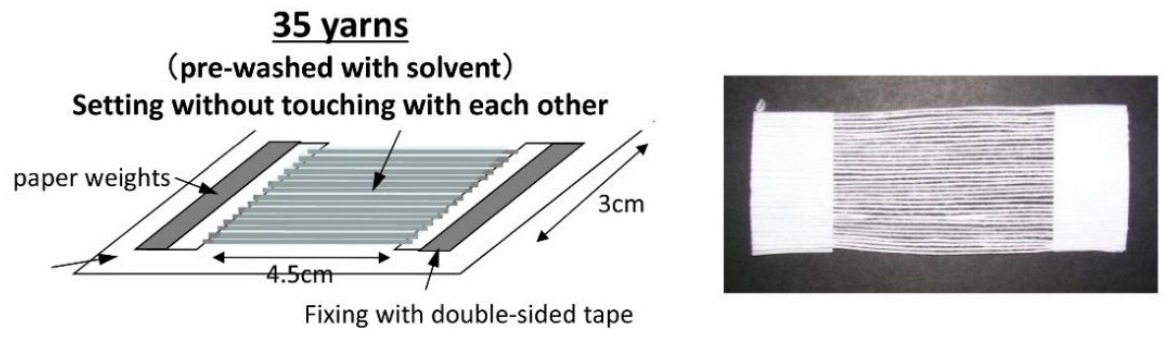

Figure 3. Sample preparation

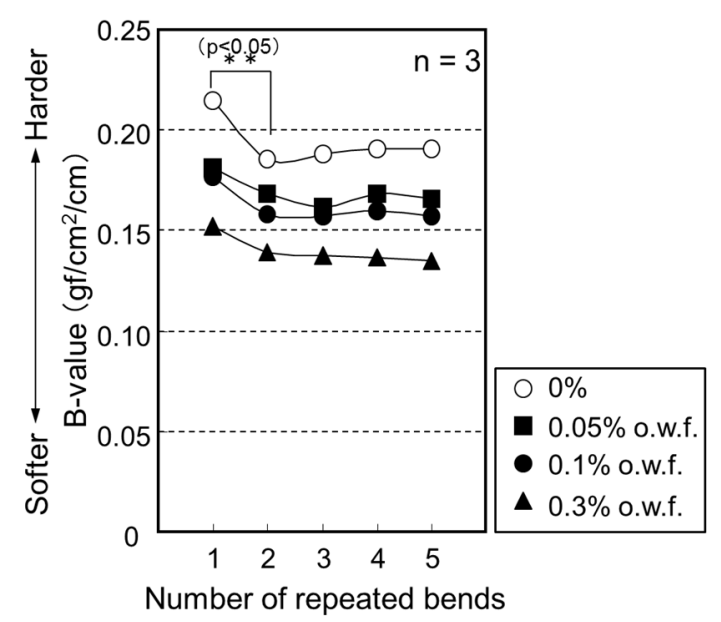

Figure 4. Results of the bending test

We think that the underlying factors that reflect the magnitude of this B-value are cross-links generated between single fibers within the threads or, more specifically, cross-links between single fibers due to hydrogen bonds mediated by bound water, because the stiffness is lost when the thread is complete-dried (described later). The significant changes observed in the B-value caused by the initial bending operation can be interpreted as a phenomenon corresponding to the destruction of the cross-linked structure through the application of the physical force for bending. To confirm this possible involvement of water molecules, we conducted the same investigation using fibers without hydroxyl groups and with low standard moisture regain, such as polyester (Shimazaki,2009), and found that the B-values of (1) and (2) consistently remained almost unchanged, irrespective of the softener treatment conditions. 


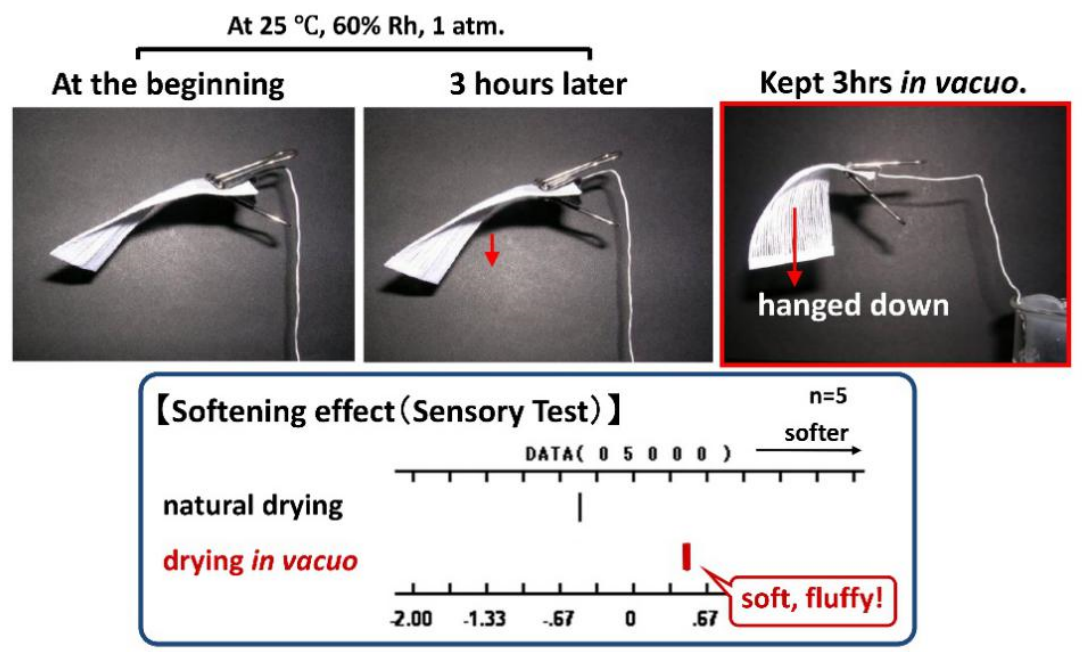

Figure 5. Changes after the Treatment in vacuo
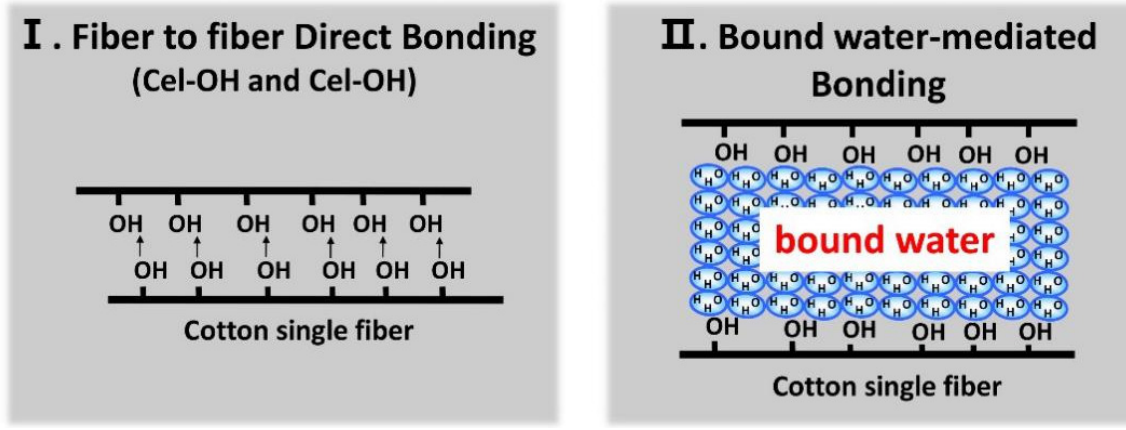

Figure 6. The image of two types of hydrogen bonding between the fibers

Based on the above information, the latter recovered B-values observed for the cotton threads can be interpreted as results that reflect the additional stiffness applied to the threads through the reforming of the hydrogen bonds formed between single fibers. Incidentally, the reason for that there is almost no recovery in the stiffness reduction associated with the initial bending operation of cotton threads is thought to be because the hydrogen bonds formed during natural drying are formed in the presence of the meniscus force (Campbell, 1947) acting between fibers; once those bonds collapse, as the distances between these single fibers are significantly big, and thus the bonds cannot be restored.

Then, we attempted to determine whether these hydrogen bonds were caused by direct coupling between hydroxyl groups on single fibers or actually caused by hydrogen bonds mediated by bound water (Kohata, Miyagawa, Takaoka, \& Kawai, 1986). We fixed one end of the samples shown in Figure 3 and confirmed that there was almost no drooping at the other end of the threads, even when it was left at room temperature and atomosphere for three hours. We then attempted to adjust the absolute drying conditions of the samples by placing them in a desiccator and heating them to $110{ }^{\circ} \mathrm{C}$ under a reduced pressure. The results, as shown in Figure 5, showed significant drooping at the other end of the samples, and the surface of the threads appeared to be fluffy. When these same samples were cooled down to room temperature under drying conditions, a sensory evaluation was conducted immediately after, and the softness was found to have significantly increased. These results suggest that the cause of the additional stiffness that occurs on cotton after natural drying may not be mainly due to the direct coupling between hydroxyl groups on single fibers (Figure 6, I), but instead mainly be due to the three dimensional bonds among single fibers mediated by bound water (cross-linking) (Figure 6, II). This finding is considered to be a result that points to the importance of the cohesion force between fibers, it was previously described by Sebastian (1986) as "adhesive force."

If the mechanism behind the soft feeling associated with the use of softeners is considered based on the above results, its main component would be inhibiting the formation of a hydrogen-bond network among single fibers mediated by bound water. 


\section{Conclusion}

If we make an overview of the studies searching for the cause of the softness obtained through use of softeners, the first notable point to be made is that a vast amount of research attempts has been made to interpret the texture based on its relationship with the properties of fibers. The individual findings reported are all very interesting, but there is a lack of findings that touch upon the fundamental principles for uniformly understanding these phenomena. From this perspective, we hope that the research presented here may assist in the understanding of these principles.

In addition, as consumers continue to feel satisfied with the basic performance of a product, they will begin to demand a higher-order different dimension of comfort amenities. Therefore, we, manufacturers and suppliers of softeners need to ascertain and appropriately respond to these demands, as well as to uncover potential needs, to proactively propose impactful products. With the recent development of remarkable analytical instruments, it has become possible to accurately analyze the dissolution and adsorption state of softeners at the nano-level, as well as to design softeners based on this information. As we move forward with these attempts, the role of the suppliers of consumer goods is to skillfully integrate the latest information in the related fields of physical science, fiber science, textile engineering, Kansei/Affective engineering, human engineering, and physiological sciences, which are essential for deepening our understanding of texture, to build new fiber reforming technology that is able to meet the need of the times, and to propose new products building on the results of these ventures.

\section{Acknowledgments}

We would like to express our sincere gratitude to Tetsuya Okano, Head of the Household Research Laboratory, and Taku Mimura, Head of the Material Science Research Laboratory, Kao Corporation. I also appreciate to Kaoru Tsuji, former professor at Hokkaido University and Masayuki Takatera, professor at Shinshu University who gave me a good advice for this research.

\section{References}

Abe, M., \& Horiuchi, T. (2011). Surfactant Function Creation, Material Development and Application Technology. NTS, p. 469.

American Association of Textile Chemists and Colorists. (1978). Tech. Paper, American Dyestuff Rep, 55, 118.

Campbell, W. B. (1947). Academic aspects of paper stock preparation. Paper Trade J, 125(19), 84.

Cruzen, A. M. (1995). Study on ditallow dimethyl ammonium chloride interaction with cellulose. Journal of American Oil Chemical Society, 72, 137.

Egan, R. R. (1978). Cationic surface active agents as fabric softeners. Journal of the American Oil Chemists' Society, 55(1), 118-121.

Egawa, N. (2008). Development trend of recent fabric softener. Fragrance Journal, 12, 38-73.

Evance, W. P. (1969). Cationic fabric softeners. Chemistry and Industry, July, 893.

Farooq, A., \& Schramm, Jr. C. (2009). Fabric Softening. In Handbook of Detergents: Part E. CRC Press.

Habereder, P., \& Bereck, A. (2002). Part 2: silicone softeners. Review of Progress in Coloration and Related Topics, 32(1), 125-137.

Hawarth, P. H. (1964). J. Text. Inst., 55(2), T251.

Herrington, T. M., \& Midmore, B. R. (1984). Adsorption of ions at the cellulose/aqueous electrolyte interface. Part 1.-Charge/pH isotherms. Journal of the Chemical Society, Faraday Transactions 1: Physical Chemistry in Condensed Phases, 80(6), 1525-1537.

Igarashi, Nakamura, Morita, \& Okamoto (2016). Elucidation of softening mechanism in rince cycle fabric softeners. J. Surfact. Deterg, 19, 183-192.

Inoue, Sano, Uyama, \& Niwa (1997). Effects of softener treatment on hand,thermal and moisture transport, and wear comfort of knitted fabrics. Journal of the Society of Fiber Science and Technology, 53(6), 226-238.

Iwazawa, Umezawa, Sawada, \& Tsuji (Ed.). (2001). Handbook of Surfactants-Chemistry and Engineering. NTS, p. 929.

Katayama, M. (2003). Science of Clothing Management. Kenpakusha, p. 100.

Kawabata, S. (1982). Objective Specification of Fabric Quality, Mechanical Properties, and Performance. Textile Machinery Society of Japan. 
Kohata, K., Miyagawa, M., Takaoka, A., \& Kawai, H. (1986). Fundamental studies on the interaction between moisture and textiles:part1. Moisture sorption and deposition isotherms of cellulosic fibers. S'eni Gakkaishi, 42, T-136.

Laghlin, R. G. (1991). Fabric softening in Cationic Surfactants. Surfactant Science Series, 3.

Larrat, E. (1966). An inquiry into the influence of application conditions on softener efficiency. Am. Dye. Rep, $55(1), 30$.

Minegishi, Y., \& Arai, H. (1977), Recent studies on cationic surfactants as domestic fabric softeners. Journal of Japan Oil Chemist's Society, 26, 2, 86.

Minegishi, Y., \& Arai, H. (1977).Recent studies on cationic surfactants as domestic fabric softener. Journal of Japan Oil Chemist's Society, 26(2), 85.

Miyasaka H. (2004), Tribology in Domestic Fabric Softener. Tribologist, 50(6), 441-446.

Miyasaka, H. (2005). Recent trends in development of domestic fabric softeners. Oleoscience, 5(10), 466.

Mohammadi, M. (2006). Colloidal Stability of di-chain cationic and ethoxylated nonionic surfactant mixtures used in commercial fabric softeners. Colloids and Surface A: Physicochem. Eng. Aspects, 288, 96-102.

Morita, T. (2007). Science of Clothing Life. IK-Publishing, p. 139.

Motoyama, \& Saiuchi. (1961). Fabric Softeners. Journal of the Society of Fiber Science and Technology, 13, 7518.

Nakamura, K., Fujiwara, N., \& Kunieda, H. (1997). Adsorption of double-chain cationic surfactant on hydrophilic surface. Journal of Japan Oil Chemist's Society, 46(2), 1461.

Nichiyohin-Keshohin Shimbun. (2018, February 12). User's Voice VOL4. Fabric softener market.

Okumura, O. (1983). A study on the adsorption of dialkyldimethyl ammonium chloride. Journal of American Oil Chemical Society, 60, 1699.

Okumura, O., \& Yokoi, K. (1986). A study on the adsorption of dialkyldimethyl ammonium chloride. Journal of the Surface Science Society of Japan, 6(5), 411.

Olofsson, B. (1950). Measurement of friction between single fibers. Text. Res. J, 20, 467.

Puchta, R. (1984). Cationic Surfactants in Laundry Detergents and Laundry After treatment Aids. JAOCS, 61(2), 367.

Röder, H. L. (1953). 16-measurements of the influence of finishing agents on the friction of fiber. Am. Dye. Rep, 44(T), 247.

Rollins, M. L., \& Tripp, V. W. (1954). Optical and electron microscopic studies of cotton fiber structure. Textile Research Journal, 24(4), 345-357.

Sakai T, Matsumoto K, Inoue S, Sonoi A, Ando T, Uchihashi T, Ando T (2012). Direct Observation for Solid-supported Bilayer Formation of Vesicles and Effects Provided by Surfactant's Properties. IACIS, Sendai, Japan.

Sebastian, S. A. R. D., Bailey, A. I., Briscoe, B. J., \& Tabor, D. (1986). Effect of a softening agent on yarn pull-out force of a plain weave fabric. Text Res J, October, 604.

Seno, M., \& Tsuji, K. (1995). Surfactant Chemistry and Application. Dainippon Tosho, p. 88.

Shimazaki, K. (2009). Science of Clothing Materials. Kenpakusha, p. 124.

Shimazaki, K., \& Sasai, K. (2007). Clothes Science. Asakura Publishing Co., p. 155.

Suzawa, T., \& Yuzawa, M. (1966). Studies on Adsorption Properties and $\zeta$-Potential of Fibrous Substances in Surface Active Agent Solutions. VII. Journal of Japan Oil Chemists' Society, 15(1), 20-26.

Takeuchi, H. (1999). Surfactants-Basic knowledge to master use of surfactants. Sangyo Tosho, p. 20.

Stock, C. R., \& Hvizdak, A. (1953). Tactual and Instrumental Ranking of Softeners on Starched and Unstarched Cottons. Text Res J, 1, 186.

\section{Copyrights}

Copyright for this article is retained by the author(s), with first publication rights granted to the journal.

This is an open-access article distributed under the terms and conditions of the Creative Commons Attribution license (http://creativecommons.org/licenses/by/4.0/). 